\title{
SIMULAÇÃO DE MULTIDÕES EM SITUAÇÕES DE RISCO IMINENTE
}

\section{CROWD SIMULATION IN IMMINENT RISK SITUATIONS}

\author{
Lucas Paes da Costa ${ }^{1}$; Danillo Roberto Pereira ${ }^{2}$; Helton Molina Sapia ${ }^{3}$ \\ Universidade do Oeste Paulista - UNOESTE \\ E-mail: ${ }^{1}$ Icpaes16@gmail.com; ${ }^{2}$ danilopereira@unoeste.br; ${ }^{3}$ helton@unoeste.br
}

RESUMO - Existem muitos métodos de simular uma multidão, todos com suas vantagens e desvantagens. Com o aumento de situações de risco iminente em grandes metrópoles, como incêndios e atentados terroristas, se faz necessário estudar o comportamento das pessoas nesse tipo de situação. O objetivo deste trabalho é desenvolver um modelo matemático-computacional para simular multidões em situações de incêndio, a fim de estabelecer relações entre as variáveis encontradas nestes cenários. 0 modelo utiliza o conceito instintivo de multidões em situações com altos níveis de stress para configurar as interações entre as pessoas e o ambiente em um sistema multiagentes dinâmico, onde cada indivíduo possui capacidades cognitivas e motoras distintas, a fim de diagnosticar a segurança de um ambiente fechado de maneira mais rápida que os métodos atuais, além de estabelecer uma relação entre o nível do incêndio, número de pessoas e a porcentagem de sobreviventes, e também avaliar a possibilidade adaptação do sistema em um algoritmo de otimização de funções.

Palavras-chave: simulação de multidões; sistemas multiagentes; evacuação.

ABSTRACT - There are many methods of simulating a crowd of people, each with its advantages and disadvantages. The increasing number of risk situations in big cities, such as fires or terrorist attacks, made it important to study the behavior of individuals in this type of situation. The purpose of this work is to develop a mathematical-computational model to simulate crowds in fire situations in order to establish relationships between different variables found in those scenarios. This work uses the instinctive concept of crowds in situations with high levels of stress to configure the interactions between people and the environment in a dynamic multiagent system, where each individual presents different cognitive and motor capacities, so that the safety of a closed environment can be diagnosed quicker than the current methods, as well as establishing relation between the fire stage, number of people and the percentage of survivors of the group, and 
also evaluate the possibility of applying the agent-environment interactions for the implementation of a function optimization system. Keywords: crowd simulation; multi-agent systems; evacuation. 


\section{INTRODUÇÃO}

O número de incidentes de risco iminente em grandes centros urbanos, sejam acidentes ou ação terrorista, aumenta a cada ano (FERREIRA, 2009). Tendo em vista que aproximadamente metade da população mundial vive em aglomerados urbanos e que a maior causa de vítimas em situações como essas não é a fonte de perigo em si, como fogo ou gás, e sim a não adaptação dos indivíduos àquela situação, cujos altos níveis de stress podem ocasionar ações destrutivas (PAN et al, 2006), é importante e necessária a elaboração de sistemas capazes de simular cenários reais, de modo que seja possível compreender e estudar as reações dos envolvidos em tais incidentes, assim reduzindo as chances de fatalidades (MUSSE, 2013; TAKAHASHI et al., 2017). Para tanto, é possível notar o crescente número de pesquisas sobre evacuação de multidões (XU et al., 2014).

Um Sistema Multiagente, ou SMA, é um modelo de simulação composto por múltiplos indivíduos com comportamento autônomo que interagem com o meio ambiente e demais indivíduos para alcançar seus objetivos próprios, de modo que a interação social aconteça de maneira semelhante ao comportamento humano. Tais sistemas são úteis em vários tipos de simulação e possuem enorme aplicabilidade e adaptabilidade, uma das aplicações sendo a simulação de multidões (HÜBNER et al., 2003; XU et al., 2014).

Existem várias abordagens para a representação de uma multidão para o desenvolvimento de simuladores, cada uma com suas vantagens e desvantagens em relação às demais (ZHENG et al., 2009). Trabalhos como os de Bakar et al. (2017), Saboia (2010) e Ferreira (2009) fazem o uso de SMAs para a construção de simuladores de situações de risco iminente, cada um com uma abordagem diferente, onde os agentes interagem e trabalham em conjunto para encontrarem uma saída segura de um local em chamas.
O presente trabalho tem por objetivo desenvolver um modelo matemáticocomputacional capaz de simular multidões com agentes distintos em situações de perigo iminente em ambientes fechados, com o intuito de analisar as relações existentes entre o número de pessoas e o nível do incêndio na porcentagem de sobreviventes. O estudo também anseia contribuir no desenvolvimento de sistemas de fiscalização de segurança mais rápidos e mais compatíveis com a realidade.

Além disso, o modelo deseja detectar os padrões na movimentação dos indivíduos, de modo que estas novas informações possam ser utilizadas em estudos futuros, contribuindo para a redução de vítimas em acidentes.

\section{TRABALHOS RELACIONADOS}

Existem na literatura muitos caminhos para o desenvolvimento de um simulador de multidões. Além disso, tais vertentes podem ser combinadas em modelos híbridos, de modo que as vantagens únicas de cada caminho sejam combinadas, tornando a simulação ainda mais coerente com a realidade. A seguir serão apresentados alguns modelos, os quais serviram de base para o sistema desenvolvido neste trabalho.

Reynolds (1987) demonstrou a possibilidade de produzir comportamentos coletivos emergentes a partir de três regras simples, as quais seguem a seguinte ordem de prioridade: separação, alinhamento e coesão. Na primeira regra, o indivíduo tende uma distância mínima entre os seus vizinhos e obstáculos. Na segunda regra, tende a manter a mesma velocidade e trajetória que seus vizinhos. Por fim, na terceira regra, o indivíduo tende a se manter centralizado em relação aos demais. A Figura 1 apresenta as regras propostas por Reynolds em ordem. 
Figura 1. Regras propostas por Reynolds.

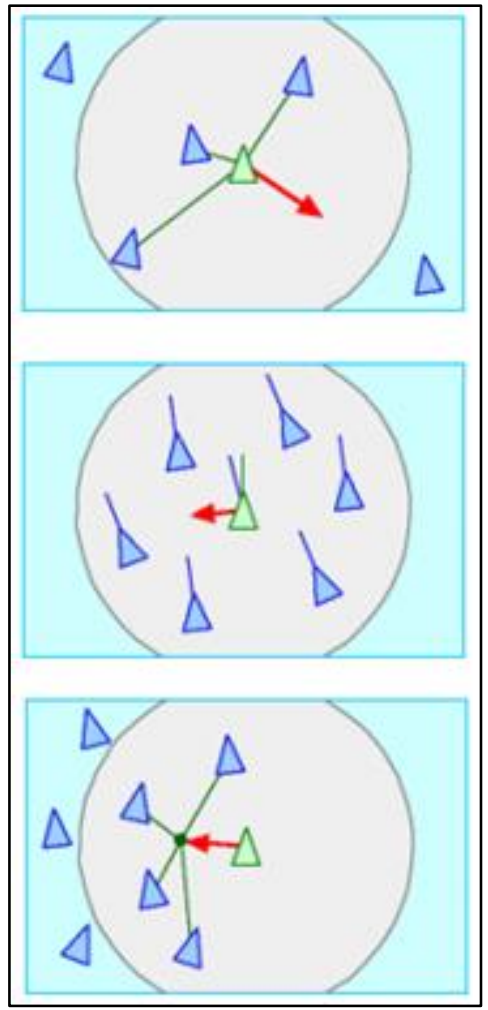

Fonte: (Reynolds, 1987).

Já o modelo de Forças Sociais, proposto por Helbing e Molnár (1995), descreve a movimentação de pedestres, cujo comportamento depende de suas experiências em situações rotineiras. A movimentação dos indivíduos é descrita por uma força nomeada Força Social, esta que representa uma somatória de forças encontradas no ambiente, sendo estas a força desejo, força psicológica e a força granular, conforme indicado na Equação 1. A primeira é um vetor direcionado ao destino do indivíduo, indicando a velocidade desejada por ele; a segunda advém da tendência de se distanciar de outros indivíduos e bordas de obstáculos, sendo uma força de interação repulsiva; a terceira, por fim, ocorre durante contato físico entre indivíduos, sendo uma força repulsiva ou dissipativa.

$$
\vec{F}_{S}=\vec{F}_{D}+\vec{F}_{P}+\vec{F}_{G}
$$

Existe ainda uma abordagem advinda da computação natural, a qual utiliza mecanismos ou comportamentos encontrados na natureza. Os modelos de simulação que aderem a essa abordagem são chamados de bioinspirados e podem ser citados como exemplo o modelo de autômato celular de von Neumann, os algoritmos genéticos e evolutivos (WOLFRAM, 1983; FRANCISCO, 2013).

É possível notar uma semelhança na representação de multidões do modelo de forças sociais com o trabalho de Reynolds (1987), sendo que tanto os pedestres quanto os grupos de animais tendem a se manter agrupados, mantendo determinada distância mínima entre indivíduos e buscando manter uma velocidade constante. Sendo assim, pode-se dizer que uma multidão segue muito mais uma postura instintiva do que racional, - que é demonstrado também pelos trabalhos de Smelser (1963) e Pan (2006).

Muitos trabalhos utilizam e adaptam os modelos relatados anteriormente para o desenvolvimento de sistemas de simulação de multidões, enquanto outros mesclam estes e outros métodos da literatura, fundindo as vantagens presentes em cada abordagem. Por exemplo, os trabalhos de Ferreira (2009) e Chennoufi et al. (2018) utilizam o modelo de Reynolds, enquanto o trabalho de Saboia (2010) faz uma mescla do modelo de Forças Sociais aliado ao modelo de Lattice-Gas, este sendo um tipo especial de autômato celular que utiliza probabilidade e estatística para estudar as características das multidões.

\section{METODOLOGIA}

O modelo desenvolvido utiliza conceitos tanto do modelo de Reynolds (1987) quanto o de Forças Sociais (HELBING; MOLNÁR, 1995), empregando uma postura instintiva nas decisões dos agentes. Essa abordagem foi escolhida com o intuito de simular pessoas sem treinamento para situações de risco iminente e algoritmo foi desenvolvido em Python.

A seguir, será apresentado um breve apanhado da literatura sobre sistemas multiagentes e a motivação das escolhas empregadas no sistema produzido neste trabalho. 


\subsection{Agente Computacional}

Segundo Ferber (1995), um agente computacional é uma entidade física ou virtual, com recursos e competências próprios, que é capaz de interagir com o meio e com outros agentes, percebendo o ambiente em que está inserido e agindo de modo a atingir seus objetivos.

Os agentes de um sistema multiagente são classificados em relação a sua constituição, podendo ser homogêneos ou heterogêneos. Um sistema cujos agentes interagem todos da mesma maneira com o ambiente em que estão inseridos é considerado homogêneo, enquanto um sistema heterogêneo possui agentes com maneiras distintas de interação com 0 ambiente (SILVA, 2003). A Figura 2 apresenta o modelo geral de um agente.

Figura 2. Modelo Geral de Agente.

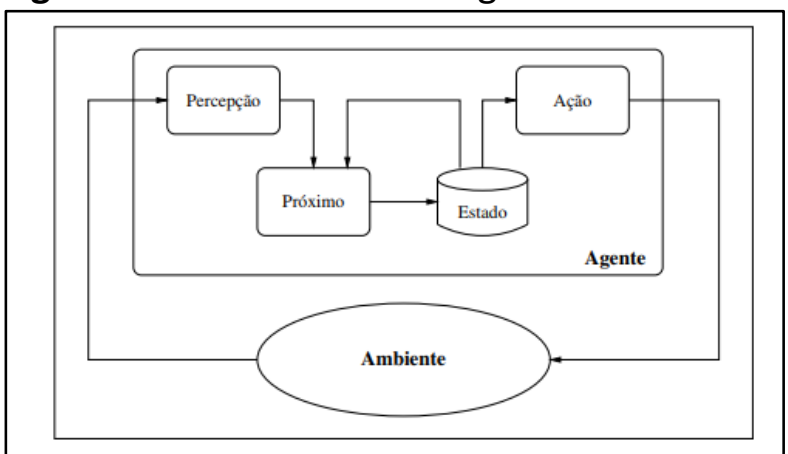

Fonte: (Hübner et al., 2018).

Além da constituição, um agente computacional ainda é classificado de acordo com sua arquitetura, esta que indica como o indivíduo percebe o ambiente e como é o seu processo de decisão. Embora existam muitas classificações na bibliografia quanto à arquitetura dos agentes, elas são distribuídas entre três classes: reativa, deliberativa ou híbrida.

Os agentes de uma arquitetura reativa são entidades simples, sem capacidade de raciocínio ou planejamento, agindo de acordo com a situação atual do ambiente em que estão inseridos, sendo incapazes de executar planos de longo prazo ou utilizar decisões anteriores na tomada de decisão futura, o que é visto como uma desvantagem da arquitetura por Reis (2003). Já como vantagem é considerada a simplicidade, desempenho computacional e grande tolerância a falhas (WOOLDRIDGE et al, 1995).

Figura 3. Exemplo de arquitetura reativa.

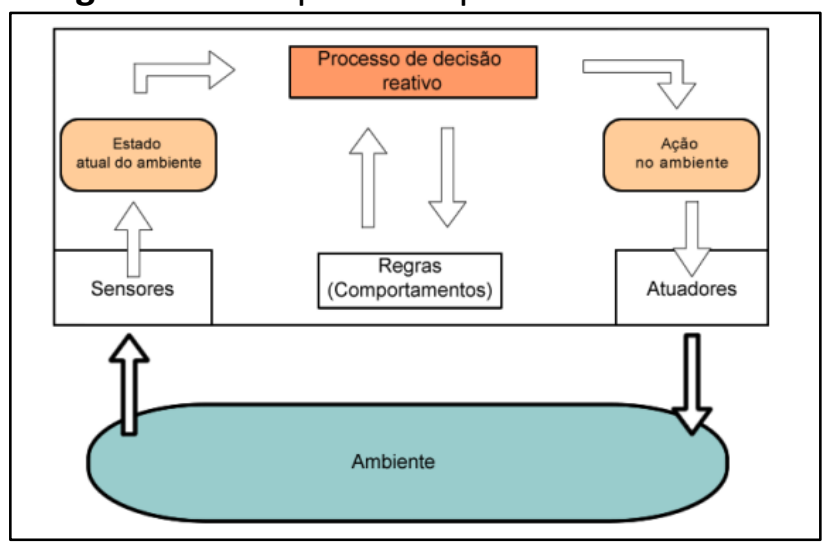

Fonte: (Ferreira, 2009).

\section{Arquiteturas}

cognitivas, ou deliberativas, seguem uma abordagem clássica de Inteligência Artificial, onde os agentes são capazes de processar informações sobre estados anteriores e sobre outros agentes. Os agentes tomam suas decisões baseadas no estado atual do ambiente e nas experiências passadas, podendo realizar planos de curta e longa duração para alcançar seus objetivos (FERREIRA, 2009). Os modelos cognitivos são caracterizados pela alta complexidade dos agentes, que são constituídos de mecanismos de inferência de decisão robustos e sofisticados, o que acaba levando pesquisadores a optar por arquiteturas alternativas (SABOIA, 2010). Um exemplo de arquitetura cognitiva é demonstrado na Figura 4. 
Figura 4. Exemplo de arquitetura cognitiva.

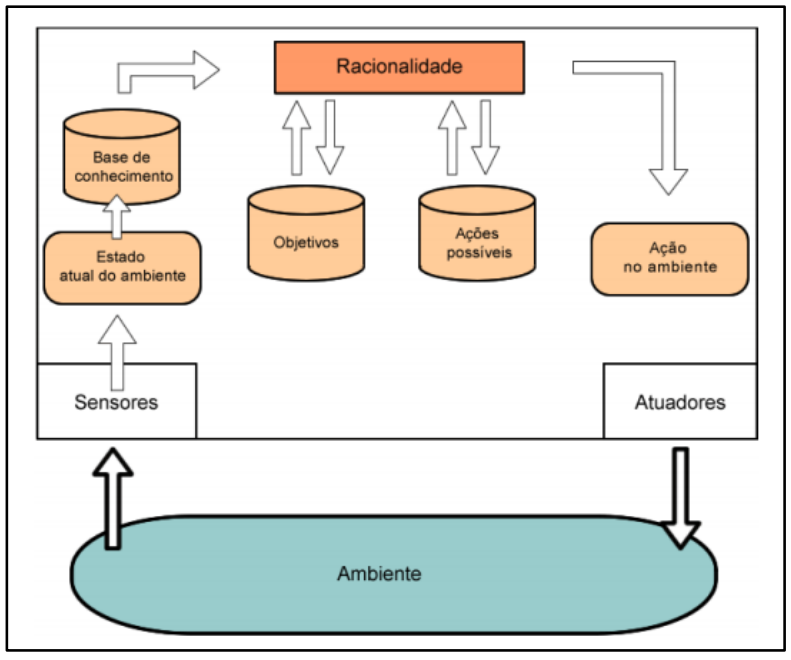

Fonte: (Ferreira, 2009).

A arquitetura híbrida, por sua vez, combina elementos de ambas as arquiteturas. Elas geralmente possuem uma hierarquia entre os comportamentos reativos e cognitivos dos agentes, de modo que os indivíduos possam responder de forma mais rápida às situações simples, típicas de uma arquitetura reativa, e possam utilizar as informações de ambientes conhecidos para situações mais complexas, otimizando o tempo de resposta em determinados estados do ambiente (FERREIRA, 2009).

Como o sistema desenvolvido objetivava a verossimilhança, as pessoas precisam apresentar capacidades físicas e cognitivas distintas, de forma que o modelo de agente homogêneos se mostra inviável. Sendo assim, o modelo utiliza a abordagem heterogênea para os agentes computacionais, onde os valores relacionados às capacidades físicas e cognitivas dos agentes obtidos aleatoriamente em distribuições normais, com média e variância de acordo com os valores encontrados em situações reais, estes obtidos através da pesquisa realizada, de modo a simular um grupo real de indivíduos.

O modelo desenvolvido também emprega uma arquitetura híbrida, onde o agente apresenta um comportamento reativo à situação atual do mesmo em relação ao ambiente e demais agentes, todavia no processo de decisão do agente é aplicado um processo de Markov, este que é um processo estocástico onde a próxima iteração é dependente do resultado das anteriores (CLARKE; DISNEY, 1979). Dessa forma, o próximo movimento de um indivíduo depende não só de seu estado atual como também de suas decisões anteriores, de maneira que o agente mantenha o caminho escolhido até que este não se mostre mais satisfatório. O método de interação será descrito com maiores detalhes na subseção dedicada ao mesmo.

O modelo de agente desenvolvido é carregado com as seguintes informações: raio de visão, velocidade, posição atual e as tendências de interação do agente.

\subsection{Ambiente de simulação}

Em simulações de evacuação de multidões é necessária a elaboração de um meio em que os agentes possam interagir para alcançar seus objetivos. É importante que o meio tenha a capacidade de dar o suporte necessário para a interação dos indivíduos (BRAUN et al., 2005). Muitos trabalhos consultados, como os de Ferreira (2010) e Pechelano et al. (2005, 2006), utilizam um ambiente bidimensional com um sistema de coordenadas para a representação de cômodos limitados por paredes e portas. A Figura 5 apresenta um exemplo de simulação de evacuação bidimensional.

Figura 5. Ambiente bidimensional.

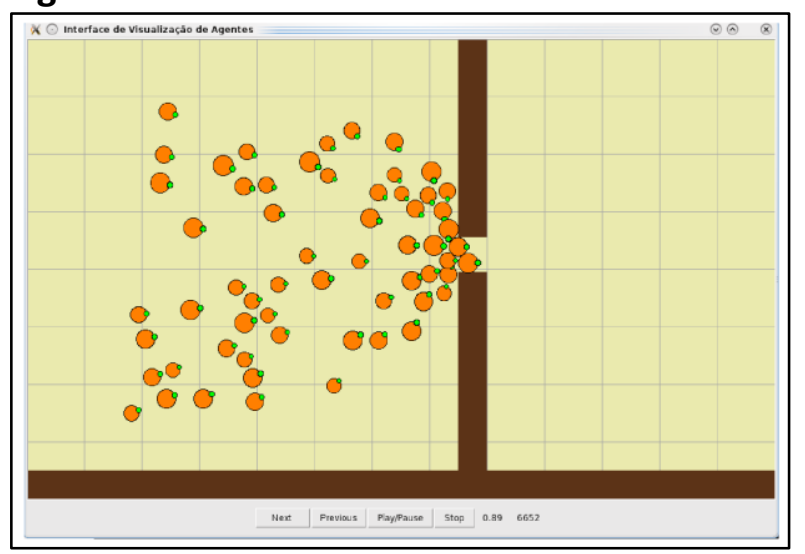

Fonte: (Saboia, 2010).

No ambiente de um sistema simulador de evacuação, os obstáculos podem ser estáticos ou dinâmicos. No 
primeiro caso, o fogo não se propaga ou diminui com o passar do tempo, nem os escombros podem ser movidos de um lugar para outro. Pode ser citado o MACES (MultiAgent Communication for Evacuation Simulation - Sistema Multiagente com comunicação para simulações de evacuação) de Pechelano et al. $(2005,2006)$ como um modelo de ambiente estático. No segundo caso, aplica-se a dinamicidade nos obstáculos, onde o fogo passa a se propagar com o tempo e há a possibilidade de remoção de escombros ou quaisquer obstáculos, podendo assim liberar possíveis saídas. O trabalho de Ferreira (2009), nomeado MACES-D, adiciona a dinamicidade ao ambiente utilizado no modelo proposto por Pechelano et al. (2005, 2006).

No modelo desenvolvido, o ambiente utiliza os conceitos de Braun et al. (2005), para a construção de um ambiente geométrico bidimensional, onde as salas são limitadas por paredes e portas e os agentes terão por objetivo encontrar a saída dos cômodos, mantendo distância dos perigos como obstáculos ou fogo. Para uma melhor representação da realidade, foi optado pela utilização de um ambiente dinâmico, onde o fogo se propaga ao longo do tempo.

\subsection{Interações entre agente e ambiente}

A interação dos agentes com o ambiente, como dito anteriormente, se dá de maneira instintiva ou reativa e acontece da seguinte forma: a pessoa observa o que existe a sua volta e toma uma decisão a partir das distâncias entre ela e os demais componentes do sistema em seu campo de visão (obstáculos, outras pessoas e saídas) e de uma tendência de decisão inicial, definida a partir do que foi levantado pelos estudos do comportamento das pessoas em situações reais. Como foi constatado em tais estudos, as pessoas tendem a formar grupos, procurar saídas e se manter distantes do fogo, ainda, as pessoas podem sofrer com os altos níveis e stress e realizar ações impensadas, ou agir em pânico.
A probabilidade de decisão inicial adotada no modelo segue esses padrões, e a pessoa então tenderá a manter uma movimentação semelhante com a observada em situações reais. Ainda no processo de decisão, foi adicionado um processo de Markov, no qual a pessoa tende a manter a decisão inicial caso se mostre satisfatória, isto é, caso uma pessoa escolha se movimentar na direção de uma outra pessoa, esta, em seu próximo movimento, terá uma tendência maior de se movimentar em direção aquela mesma pessoa se isso mantiver o primeiro agente distante $o$ suficiente dos focos de fogo ou obstáculos. Tanto a velocidade quanto a percepção da pessoa são determinadas aleatoriamente através de distribuições normalizadas, de modo a manter a verossimilhança.

Os testes foram realizados em uma sala quadrada de 50 metros de comprimento e com duas portas, uma no centro da parede ao norte e uma no centro da parede ao sul. As partículas que representam pessoas, focos de incêndio e obstáculos são posicionados aleatoriamente na sala, e o objetivo dos agentes é chegar em uma das saídas. As partículas de fogo possuem uma probabilidade de $5 \%$ de gerarem outra partícula de fogo, o que faz com que a sala seja tomada em pouco alguns minutos, assim como é observado em casos reais. A Figura 6 apresenta as imagens da simulação geradas pelo sistema desenvolvido, sendo as pessoas, o fogo, os escombros, as paredes e portas representadas respectivamente pelas cores branca, vermelha, azul, verde e verde claro. 
Figura 6. Representação visual da simulação.
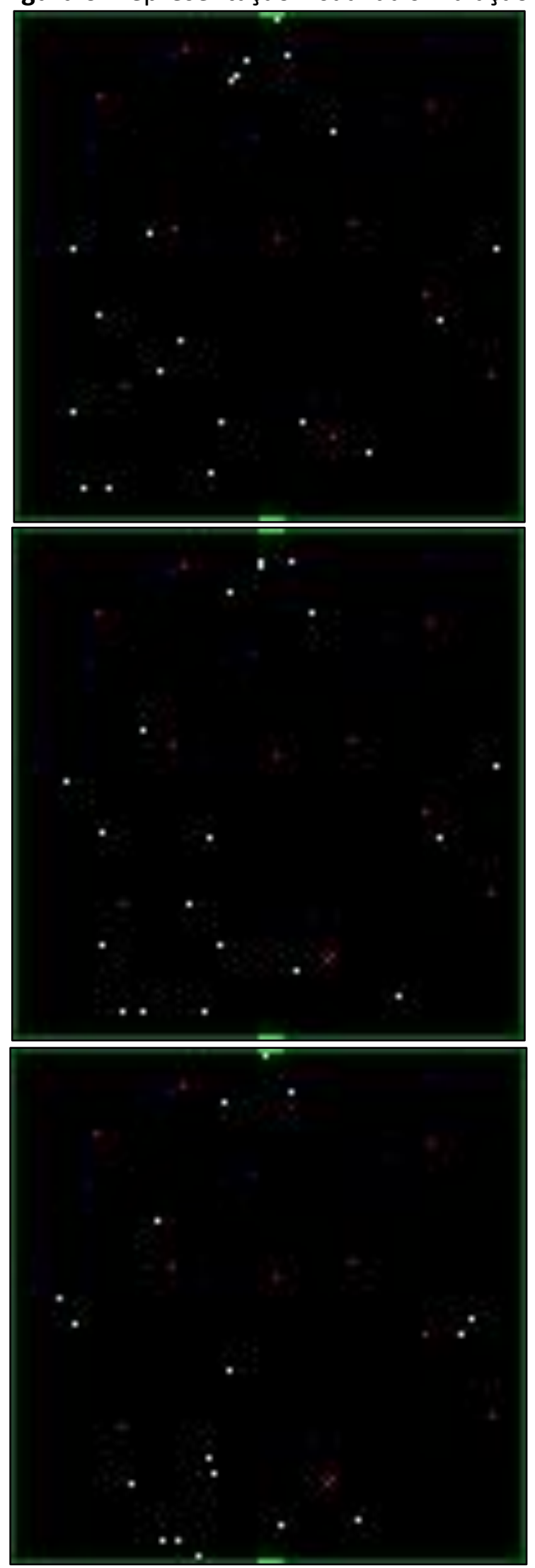

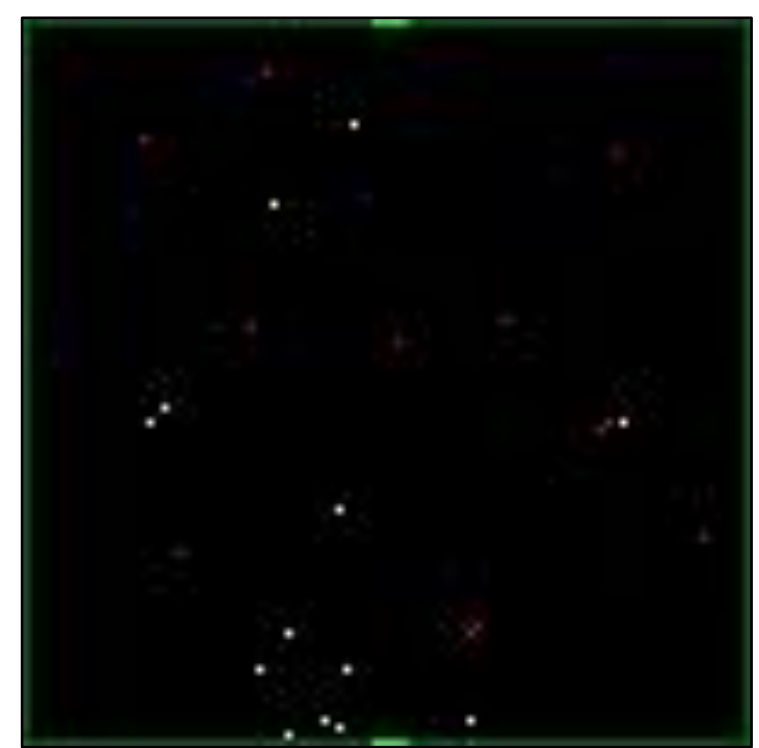

Fonte: Os autores.

É possível observar na Figura 6 a movimentação das pessoas e a formação de grupos. Também pode ser observada a propagação do fogo, ainda que sendo pouca, já que o período entre o primeiro e último quadro da imagem é de oito segundos. A Figura 7 apresenta o estado do incêndio na sala após dois minutos.

Figura 7. Estado do incêndio após dois minutos.

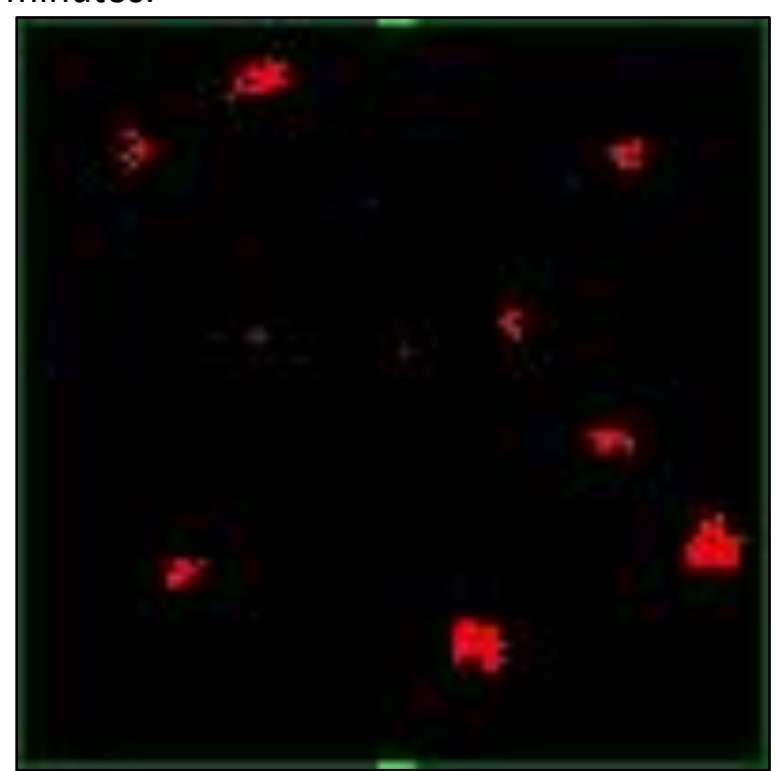

Fonte: Os autores.

O processo de decisão se dá conforme a Figura 7. O agente verifica as distâncias entre ele e as partículas mais próximas. Caso a saída mais próxima esteja a 4 metros ou menos de distância do indivíduo, ele correrá 
para a saída e fugirá do incêndio. Caso o fogo mais próximo dele não esteja a pelo menos 1,5 metros de distância, ele imediatamente sairá de perto da partícula de fogo, com direção contrária ao vetor distância entre eles. Caso ele esteja a menos de 10 metros de uma saída, ele ignorará suas tendências e correrá para a saída. Todavia, se nenhum dos casos apontados acontecer, o agente se comportará de acordo com as tendências instintivas determinadas pelo sistema de simulação. Independentemente da decisão do agente, a probabilidade de ele escolher o mesmo caminho novamente aumentará, tornando a decisão dele um processo de Markov onde cada decisão do agente altera a probabilidade das demais, de modo a simular que a pessoa tende a manter sua decisão, uma vez que cada iteração observada corresponde a um segundo de tempo.

Figura 7. Processo de decisão do agente.

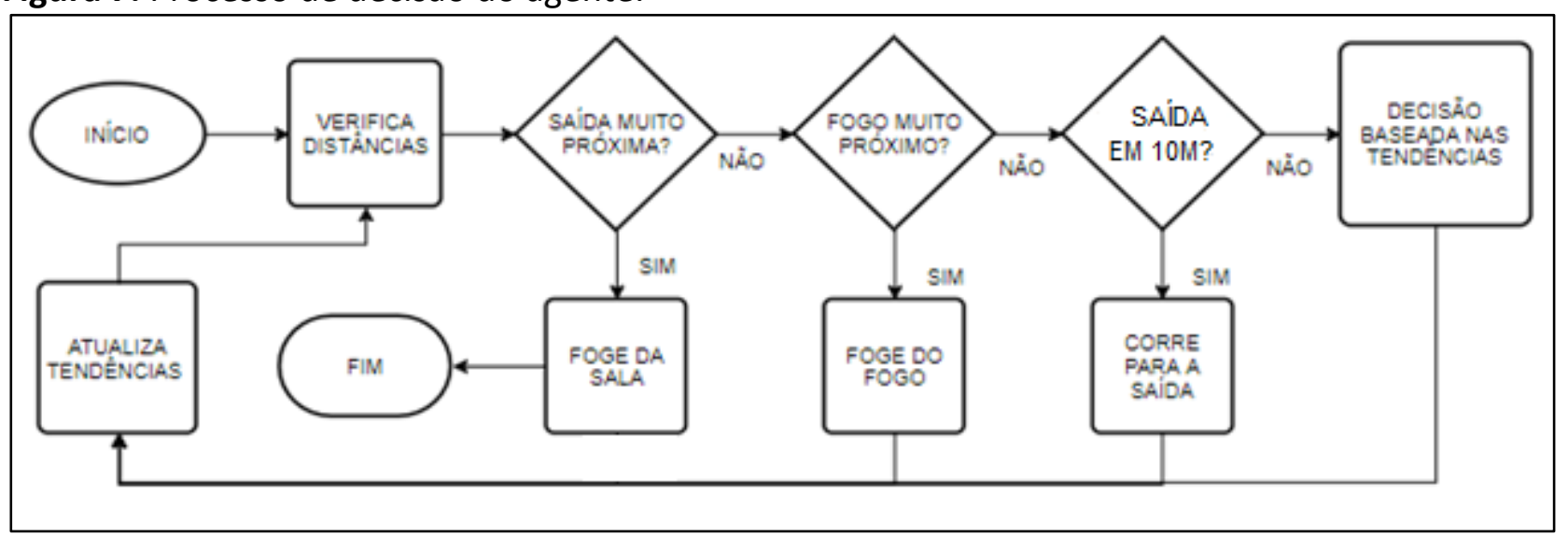

Fonte: Os autores.

Foram realizadas simulações com diferentes quantidades de pessoas, princípios de incêndio e obstáculos com o intuito de estabelecer relações entre tais variáveis com a porcentagem de sobreviventes ao incidente. Cada amostra indicava a razão de pessoas que conseguiram escapar da sala pelo número total de pessoas no início da simulação nos primeiros dois minutos de simulação, considerando que após esse tempo a situação da sala já estava crítica. Foram coletadas 100 amostras para cada combinação de quantidade de pessoas, focos de incêndio e obstáculos.

\section{RESULTADOS E DISCUSSÃO}

O Gráfico 1 apresenta a progressão encontrada das médias e desvios padrão de acordo com o número de pessoas na simulação e o nível de incêndio, sendo o primeiro nível de incêndio uma situação onde o incêndio está em seu início e a terceira uma situação onde o incêndio já está bem espalhado pela sala, enquanto a segunda combinação é uma situação intermediária entre as outras duas. Em todos os casos, os agentes apresentaram um tempo médio de saída 18 e 28 segundos, com desvios padrão variando entre 13 e 17 segundos.

Gráfico 1. Progressão das médias e desvios padrão da porcentagem de sobreviventes.

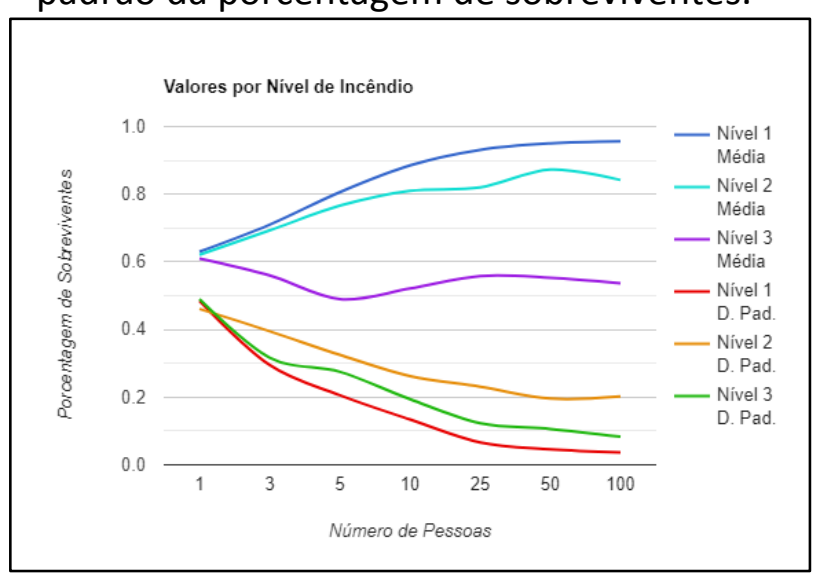

Fonte: Os autores. 
Analisando a progressão apresentada no Gráfico 1, é possível observar que a porcentagem média de sobreviventes aumenta até estabilizar em determinado ponto para os dois primeiros níveis de incêndio, bem como o desvio padrão tende a reduzir para todos os casos. Já para a terceira combinação, cujo incêndio já se inicia com maior intensidade, se observou que o valor médio de porcentagem oscila nas proximidades de $50 \%$.

A redução numérica do desvio padrão significa resultados mais confiáveis e concisos em relação à média encontrada. Como o valor da média se mantém crescente, ou ao menos estável em valores altos, isso significa que as chances de sobrevivência se tornam mais amigáveis, uma vez que existe uma tendência maior onde a maioria das pessoas consiga escapar do incêndio.

Outra relação que pode ser observada é a relação entre a porcentagem de sobreviventes e o nível do incêndio, já que o Gráfico 1 mostra a redução na média de sobreviventes para os níveis 2 e 3 . Tal fenômeno ocorre, pois os indivíduos tendem a se direcionar pela movimentação das demais pessoas em seu campo de visão, além de evitar se aproximar dos obstáculos. Isso se torna uma abordagem positiva para situações onde o incêndio ainda não está agravado, pois existem muitos caminhos seguros até a saída. Todavia, nos casos onde o incêndio já está muito espalhado pela sala, como na terceira combinação, há uma possibilidade significativa do indivíduo, ao seguir determinadas pessoas, acabar se afastando das saídas e ser encurralado pelas chamas.

\section{CONSIDERAÇÕES FINAIS}

Este trabalho apresentou um sistema de simulação de multidões baseado nos modelos de Reynolds e de Forças Sociais, utilizando um sistema multiagentes com arquitetura híbrida, onde as decisões passadas interferem nas relações futuras entre as pessoas e o ambiente a sua volta. Apesar da simplicidade do modelo desenvolvido, este apresentou resultados verossímeis e similares a outros modelos de simulação de evacuação.

O modelo desenvolvido apresenta a vantagem de ser facilmente implementado e apresentar verossimilhança, porém pode ser melhorado, com a adição de colisão de partículas, comunicação entre os indivíduos e maior dinamicidade nos obstáculos, como escombros deslocáveis e outros tipos de perigos, como pessoas armadas e portas emperradas ou trancadas.

Através das simulações realizadas com o modelo, é possível observar as áreas com maiores tendências de circulação de pessoas, além de estabelecer as melhores rotas de evacuação para as mesmas, de forma a facilitar o estudo para adequação de ambientes.

O sistema de simulação também pode ser adaptado em relação à sua aplicabilidade, sendo desenvolvida uma interface para usuários, com a possibilidade de ler plantasbaixas de ambientes reais, de modo que se torne mais rápida e prática a análise de segurança realizada pelo Corpo de Bombeiros.

Ainda, o comportamento de multidões em situações de risco iminente se mostrou generalizável, podendo então ser aplicado à otimização de funções baseado em algoritmos bioinspirados, como a otimização baseada em colônia de formigas ou por enxame de partículas, sendo esta também uma vertente para trabalhos futuros.

\section{REFERÊNCIAS}

BAKAR, N. A. A. et al. A simulation model for crowd evacuation of fire emergency scenario. In: INTERNATIONAL CONFERENCE ON INFORMATION TECHNOLOGY (ICIT). 8., Amman. $2017 . \quad$ p. 361-368. https://doi.org/10.1109/ICITECH.2017.80800 $\underline{27}$

BRAUN, A.; BODMANN. B. E. J.; MUSSE, S. R. Simulating virtual crowds in emergency 
situations. [S.I.]: ACM. 2005. p. 244-252. https://doi.org/10.1145/1101616.1101666

CLARKE, A. B.; DISNEY, R. L. Probabilidade e processos estocásticos. 1. ed. Rio de Janeiro: Livros Técnicos e Científicos, 1979.

FERBER, J. Les Systèmes multi-agents: Vers une intelligence collective. [S.I.]: InterEditions, 1995.

FERREIRA, Y. S. Um estudo investigativo sobre a comunicação em simulações de emergência e evacuação. 2009. Dissertação (Mestrado em Computação) - Universidade Federal Fluminense. Niterói, 2009

FRANCISCO, D. O. Aplicação de algoritmos bio-inspirados ao problema de geração automática de grades horárias. 2013. Dissertação (Mestrado em Ciências) - Escola de Engenharia de São Carlos. Universidade de São Paulo. São Carlos. 2013.

HELBING, D.; MOLNÁR, P. Social force model for pedestrian dynamics. Physical Review E (Statistical Physics, Plasmas, Fluids, and Related Interdisciplinary Topics), v. 51, n. 5, p. 4282-4286, May, 1995. https://doi.org/10.1103/PhysRevE.51.4282

HÜBNER, J. F.; SICHMAN, J. S. Organização de sistemas multiagentes. Anais Congresso da Sociedade Brasileira de Computação. v. 8, n. 23, p. 247-296, 2003.

HÜBNER, J.; BORDINI, R.; VIEIRA, R. Introdução ao desenvolvimento de sistemas multiagentes com Jason. 2018

MUSSE, S. R. Simulação de Multidões de Humanos Virtuais. Youtube. 3 ago. 2013. Disponível em <http://hdl.handle.net/10923/5978>. Acesso em 20 abr. 2018.

PAN, $X$. et al. Human and social behavior in computational modeling and analysis of egress. Automation in construction, v. 15, p.
448-461, 2006. https://doi.org/10.1016/j.autcon.2005.06.00 $\underline{6}$

PELECHANO, N. et al. Crowd Simulation Incorporating Agent Psychological Models, Roles and Communication. [S.I.]: [s.n.]. 2005. p. 21-30.

https://doi.org/10.21236/ADA522128

PELECHANO, N.; BADLER, N. I. Modeling Crowd and Trained Leader Behavior during Building Evacuation. IEEE Comput. Graph. Appl., v. 26, p. 80- 86, 2006. https://doi.org/10.1109/MCG.2006.133

REIS, L. P. Coordination in Multi-Agent Systems: Applications in University Management and Robotic Soccer. FEUP. [S.I.]. 2003.

REYNOLDS, C. W. Flocks, Herds, and Schools: A Distributed Behavioral Model. Computer Graphics, v. 21, p. 25-34, 1987. https://doi.org/10.1145/37402.37406

SABOIA, P. C. Simulação de Multidões com Agentes Brownianos e Modelo de Forças Sociais Modificado. 2010. Dissertação de mestrado. Instituto de Computação UNICAMP, Campinas.

SILVA, L. A. M. Estudo de Desenvolvimento de Sistemas Multiagentes usando Jade: Java Agent Development Framework. Trabalho de Conclusão de Curso (Graduação em Ciências da Computação) - Centro Tecnológico. Universidade Federal de Pernambuco, Pernambuco. 2003.

SMELSER, N. J. Theory of Collective Behavior. The Free Press. New York. 1963. https://doi.org/10.1037/14412-000

TAKAHASHI, T.; ICHINOSE, K. Crowd control and evacuation guidance based on simulations. 2017 IEEE International Conference on Big Data (Big Data). Boston. 2017. 
https://doi.org/10.1109/BigData.2017.82583

$\underline{76}$

WOLFRAM, S. Statistical mechanics of cellular automata. Revisions of Modern Physics, 55(3):601-644. jul. 1983. https://doi.org/10.1103/RevModPhys.55.601

WOOLDRIDGE, M.; JENNINGS, N. R. Agent theories, architectures, and languages: a survey. [S.I.]: Springer-Verlag New York, Inc. 1995. p. 1-39. https://doi.org/10.1007/3-540$\underline{58855-81}$

$X U, M$. et al. Crowd Simulation and Its Applications: Recent Advances. Journal of Computer Science and Technology. 29(5). p. 799-811. Set. 2014. https://doi.org/10.1007/s11390-014-1469-y

ZHENG, X.; ZHONG, T.; LIU, M. Modeling crowd evacuation of a building based on seven methodological approaches. Building and Environment. v. 44, p. 437-445, 2009. https://doi.org/10.1016/i.buildenv.2008.04.0 $\underline{02}$ 